\title{
Association of Newer Definitions of Bronchopulmonary Dysplasia with Pulmonary Hypertension and Long-Term Outcomes
}

\section{Jae Kyoon Hwang ( $\nabla$ jkhwang@hanyang.ac.kr)}

Hanyang University Guri Hospital https://orcid.org/0000-0003-0312-567X

\section{Seung Han Shin}

Seoul National University Children's Hospital https://orcid.org/0000-0002-7008-4073

\section{Ee-Kyung Kim}

Seoul National University Children's Hospital

\section{Seh Hyun Kim}

Seoul National University Children's Hospital

\section{Han-Suk Kim}

Seoul National University Children's Hospital

\section{Research Article}

Keywords: Bronchopulmonary dysplasia, Definition, Pulmonary arterial hypertension, Respiratory morbidity, Neurodevelopmental impairment

Posted Date: November 23rd, 2021

DOI: https://doi.org/10.21203/rs.3.rs-1078279/v1

License: (c) (1) This work is licensed under a Creative Commons Attribution 4.0 International License. Read Full License 


\section{Abstract}

The diagnostic criteria of bronchopulmonary dysplasia (BPD) have been reassessed based on current practices and highest accuracy in predicting long-term outcomes. We investigated the association between BPD severity based on different definitions in terms of long-term outcomes and pulmonary arterial hypertension (PAH). This retrospective study enrolled preterm infants born at $<32$ weeks of gestation. The definition of BPD recommended by the National Institutes of Health in 2001, National Institute of Child Health and Human Development (NICHD) in 2018, and Jensen et al. in 2019 were used. The association between re-hospitalization due to respiratory illness, neurodevelopmental impairment (NDI) at a corrected age of 18-24 months, and PAH at a postmenstrual age (PMA) of 36 weeks with the severity of BPD based on these three definitions were evaluated. Among 354 infants, gestational age ( 26.9 weeks) and birth weight $(730 \mathrm{~g})$ were lowest in severe BPD based on NIH 2001 definition. In total, $14.1 \%$ of study population experienced NDI and $19.0 \%$ were re-hospitalized due to respiratory illness. Multiple logistic regression analysis showed that the adjusted odds ratio (OR) for re-hospitalization was highest in grade-3 BPD of the Jensen (2019) criteria (7.45). The adjusted OR for NDI (13.23) and PAH (40.37) were also highest in grade-3 of the Jensen (2019) criteria.

Conclusion: Based on recently suggested criteria, severity of BPD is associated with long-term outcomes and PAH at PMA of 36 weeks in preterm infants.

\section{What Is Known}

Since BPD was first described in 1967, the definition and characteristics of the disease have evolved over the past 50 years as more immature infants can now survive. In recent years, the diagnostic criteria of BPD have been reassessed based on current practices and highest accuracy in predicting long-term outcomes.

\section{What is new}

Based on recently suggested criteria, severity of BPD is associated with long-term outcomes and PAH at PMA of 36 weeks in preterm infants.

\section{Introduction}

Bronchopulmonary dysplasia (BPD) is chronic lung disease that occurs in preterm infants and is one of the most important morbidities that determine later health outcomes in this population. Since it was first described in 1967,[1] the definition and characteristics of the disease have evolved over the past 50 years as more immature infants can now survive. So far, the definition of BPD proposed in 2001 in a consensus conference of the National Institutes of Health $(\mathrm{NIH})$ is the most commonly used, and this categorized the severity of BPD based on the mode of respiratory support and the amount of supplemental oxygen required.[2] Subsequently, a physiological definition of BPD has also been suggested as the degree of 
respiratory support is determined by each attending physician, rather than according the basis of functional assessment.[3]

However, the limitations of the definitions of BPD have not yet been completely addressed.[4] One of the most important issues is the evolution of various modes of respiratory support in preterm infants over the last decades, which trends towards less invasive respiratory support strategies in order to minimize lung injury in patients with immature lungs.[5; 6] In this context, revised refinements of the definition of BPD were suggested in the National Institute of Child Health and Human Development (NICHD) workshop in 2018, which considered non-invasive positive pressure ventilation (NIPPV) and humidified high flow nasal cannula (HHFNC) as important modalities.[7] More recently, Jensen et al. explored various criteria for diagnosing BPD in view of current practices in terms of the ability to accurately predict later health outcomes.[8]

Another critics have pointed out that the etiology and pathophysiology of the disease were not considered in the BPD criteria in 2001.[4] Pulmonary vascular disease has been recently recognized as an important pathophysiology of BPD in neonates born with immature lungs, and the association of BPD with this condition has been demonstrated as there is a relatively high prevalence of pulmonary arterial hypertension (PAH) in patients with severe BPD. [9; 10] Even though it is difficult to include PAH in the current definition of BPD, a better-categorized definition of BPD may identify the association of PAH more clearly in severe forms of the disease.

In this study, three criteria for diagnosing BPD in preterm infants, including the NIH 2001, NICHD 2018, and Jensen's (2019) criteria, were used to predict respiratory and neurodevelopmental outcomes at a corrected age (CA) of 18-24 months. Furthermore, the association of BPD with PAH at a post menstrual age (PMA) of 36 weeks was explored using each criterion.

\section{Methods}

\section{Population and Data Source}

This retrospective study enrolled preterm infants who were born at $<32$ weeks' gestation at Seoul National University Hospital, between January 2014 and December 2018. Infants with congenital anomalies or congenital infections and infants who died before being discharged were excluded from the study population. Those who were lost to follow-up at a CA of 18-24 months and those who died after being discharged before a CA of 18-24 months were also excluded. Data on perinatal characteristics, clinical courses, PAH, and mode of respiratory support at a PMA of 36 weeks were collected. PAH was diagnosed using echocardiography based on the following findings: right-to-left or bidirectional shunt, velocity of tricuspid regurgitation $\geq 3 \mathrm{~m} / \mathrm{s}$, and left-deviated or flat configuration of the interventricular septum. [11] Re-hospitalization due to respiratory illness until a CA of 18-24 months and neurodevelopmental outcomes at a CA of 18-24 months were evaluated. This study was approved by the Institutional Review Board of Seoul National University Hospital (2109-008-1250). Obtaining informed consent was waived by 
the board and all methods were performed in accordance with the guidelines of the Human Research Protection Program.

\section{Grading severity of the BPD based on the NIH 2001, the NICHD 2018, and the Jensen's (2019) criteria}

First, BPD was diagnosed and graded as mild, moderate and severe disease according to the definition established by the NIH in 2001 (NIH 2001). [2] Second, the recently proposed skeletal definition of BPD by NICHD in 2018 was used (NICHD 2018) (See supplementary material, Table S1).[7] In 2019, Jensen et al. compared a number of diagnostic criteria for BPD, in terms of various type of non-invasive respiratory support.[8] Among the criteria, those that most accurately predicted later health outcomes were adopted in this study. These were used to categorize BPD that requires respiratory support with low-flow ( $\leq 2$ $\mathrm{L} / \mathrm{min}$ ) nasal cannula at PMA of 36 weeks as grade 1; BPD that requires high flow ( $>2 \mathrm{~L} / \mathrm{min}$ ) or nasal continuous positive airway pressure or NIPPV at PMA of 36 weeks as grade 2; and BPD that requires invasive positive pressure ventilation at PMA of 36 weeks as grade 3 (Jensen 2019) (Table S1).

\section{Outcomes}

Respiratory morbidity was defined as re-hospitalization due to respiratory illness until a CA of 18-24 months. The Bayley Scales of Infant and Toddler Development $3^{\text {rd }}$ Edition (Bayley-III) results at a CA of 18-24 months were reviewed and scores of $<85$ (-1 SD) in both cognitive and language domains or a motor score of $<85$ were defined as developmental delay. [12] Combined neurodevelopmental impairment (NDI) was defined when there was any of following: blindness, hearing impairment that required the use of hearing aids, cerebral palsy, and developmental delay in Bayley-III.

\section{Statistical Analyses}

Fisher's exact test was used for categorical variables, and one-way analysis of variance was conducted to compare continuous variables according to the severity of BPD using the NIH 2001 criteria. Gestational age, birth weight z-score, and sex were adjusted in the multivariate logistic regression analysis for later respiratory and neurodevelopmental outcomes as well as PAH at a PMA of 36 weeks based on the three different definitions of BPD. Values were expressed as $\mathrm{n}(\%)$ or median (interquartile range), and a $\mathrm{P}$ value $<0.050$ was considered to indicate statistical significance. STATA 12.0 software for Windows (Stata Corp., College Station, TX, USA) was used to analyze all data.

\section{Results}

During the study period, 479 infants who were $<32$ weeks' gestation were born at our facility. Nineteen babies with congenital anomalies, one baby with congenital cytomegalovirus infection, and 60 babies who died before they were discharged were excluded (Figure 1). Among the 399 infants who survived until they were discharged, one died after being discharged and 45 were lost to follow-up at a CA of 1824 month, the remaining 354 infants were included in the final analysis. 
Demographic findings of the study population were summarized according to the severity of BPD based on the NIH 2001 criteria (Table 1). Gestational age (26.9 weeks (25.3-29)) and birth weight (730 grams (620-980)) were lowest in infants with severe BPD, and the incidence of small for gestational age was highest in the group of infants with severe BPD $(20.8 \%)$. While the prevalence of multiple births was highest in infants without BPD (74.3\%), histologic chorioamnionitis and oligohydramnios were most common in the severe BPD group ( $62.3 \%$ and $35.8 \%$, respectively). The prevalence of respiratory distress syndrome, PDA requiring treatment, high-grade IVH, ROP operation, and pulmonary hypertension at a PMA of 36 weeks were highest in infants with severe BPD (Table 2).

Table 1

Demographics of the study population according to the severity of bronchopulmonary dysplasia (NIH 2001)

\begin{tabular}{|c|c|c|c|c|c|}
\hline & $\begin{array}{l}\text { No BPD } \\
(n=202)\end{array}$ & $\begin{array}{l}\text { Mild BPD } \\
(n=67)\end{array}$ & $\begin{array}{l}\text { Moderate BPD } \\
(n=32)\end{array}$ & $\begin{array}{l}\text { Severe BPD } \\
(n=53)\end{array}$ & $\begin{array}{l}\mathrm{p}- \\
\text { value }\end{array}$ \\
\hline GA (week) & $30.7(30-31.3)$ & $\begin{array}{l}27.6(26.6- \\
28.6)\end{array}$ & $\begin{array}{l}28.2(26.4- \\
29.5)\end{array}$ & $\begin{array}{l}26.9(25.3- \\
29)\end{array}$ & $<0.001$ \\
\hline Birth weight (g) & $\begin{array}{l}1380(1230- \\
1590)\end{array}$ & $\begin{array}{l}1000(850- \\
1170)\end{array}$ & $\begin{array}{l}990(790- \\
1220)\end{array}$ & $\begin{array}{l}730(620- \\
980)\end{array}$ & $<0.001$ \\
\hline $\begin{array}{l}\text { Birth weight z- } \\
\text { score }\end{array}$ & $-0.2(-0.6-0.3)$ & $0.1(-0.3-0.5)$ & $-0.1(-1-0.5)$ & $\begin{array}{l}-0.3(-1.1- \\
0.3)\end{array}$ & 0.021 \\
\hline SGA & 16 (7.9) & $3(4.5)$ & $6(18.8)$ & $11(20.8)$ & 0.006 \\
\hline Male & $101(50)$ & $35(52.2)$ & $20(62.5)$ & $23(43.4)$ & 0.394 \\
\hline $\mathrm{C} / \mathrm{S}$ & $114(56.4)$ & $41(61.2)$ & $16(50)$ & $35(66)$ & 0.443 \\
\hline Multiple birth & $150(74.3)$ & $40(59.7)$ & $13(40.6)$ & $24(45.3)$ & $<0.001$ \\
\hline hCAM & 67 (33.3) & $36(54.6)$ & $14(45.2)$ & $33(62.3)$ & $<0.001$ \\
\hline PROM & 78 (38.8) & $31(47)$ & $13(40.6)$ & $25(48.1)$ & 0.499 \\
\hline Oligohydramnios & $32(15.8)$ & $16(23.9)$ & $8(25)$ & 19 (35.9) & 0.013 \\
\hline Antenatal steroid & $170(84.2)$ & $57(85.1)$ & $30(93.8)$ & $50(94.3)$ & 0.150 \\
\hline
\end{tabular}


Table 2

Clinical courses according to the severity of BPD (NIH 2001)

\begin{tabular}{|llllll|}
\hline & $\begin{array}{l}\text { No BPD } \\
(\mathbf{n = 2 0 2})\end{array}$ & $\begin{array}{l}\text { Mild BPD } \\
(\mathbf{n = 6 7 )}\end{array}$ & $\begin{array}{l}\text { Moderate BPD } \\
(\mathbf{n = 3 2})\end{array}$ & $\begin{array}{l}\text { Severe BPD } \\
(\mathbf{n = 5 3 )}\end{array}$ & p-value \\
\hline RDS & $67(33.2)$ & $51(76.1)$ & $24(75)$ & $43(81.1)$ & $<0.001$ \\
\hline PDA treated & $24(17.8)$ & $32(48.5)$ & $17(54.8)$ & $35(68.6)$ & $<0.001$ \\
\hline IVH (grade $\geq 3)$ & $2(1)$ & $2(3)$ & $1(3.1)$ & $6(11.3)$ & 0.004 \\
\hline NEC & $3(1)$ & $4(3)$ & $1(4)$ & $6(1)$ & 0.007 \\
\hline ROP operation & $2(1)$ & $3(4.5)$ & $4(12.5)$ & $23(43.4)$ & $<0.001$ \\
\hline PAH at PMA 36 weeks & $1(1.4)$ & $1(1.5)$ & $3(9.4)$ & $10(18.9)$ & $<0.001$ \\
\hline $\begin{array}{l}\text { Values are expressed as n (\%) or median (interquartile range). BPD, bronchopulmonary dysplasia; NIH, } \\
\text { National Institutes of Health; RDS, respiratory distress syndrome; PDA, patent ductus arteriosus; IVH, } \\
\text { intraventricular hemorrhage; NEC, necrotizing enterocolitis; ROP, retinopathy of prematurity; PAH, } \\
\text { pulmonary hypertension; PMA, postmenstrual age }\end{array}$ & & & \\
\hline
\end{tabular}

In total, $50(14.1 \%)$ infants experienced combined NDI at a CA of 18-24 months and $67(19.0 \%)$ were rehospitalized due to respiratory illness (Table 3). Re-hospitalization due to respiratory illness was higher in the moderate and severe BPD groups (34\% and $54.6 \%$, respectively) than in infants without BPD $(14.1 \%)$. Scores of cognitive, language, and motor domains in Bayley-III were lowest in the severe BPD group compared to the other groups (90 (80-95), 84.5 (74-94) and 91 (79-97), respectively). Delays in both cognitive and language domains, and in the motor domain were highest in the severe BPD group (23.9\% and $32.6 \%$, respectively). CP occurred most frequently in the severe BPD group (9.4\%), but there were no cases of blindness or hearing impairment that required the use of hearing aids. 
Table 3

Neurodevelopmental and respiratory outcomes according to the severity of BPD (NIH 2001)

\begin{tabular}{|c|c|c|c|c|c|}
\hline & $\begin{array}{l}\text { No BPD } \\
(n=202)\end{array}$ & $\begin{array}{l}\text { Mild BPD } \\
(n=67)\end{array}$ & $\begin{array}{l}\text { Moderate BPD } \\
(n=32)\end{array}$ & $\begin{array}{l}\text { Severe BPD } \\
(n=53)\end{array}$ & $\begin{array}{l}\mathrm{p}- \\
\text { value }\end{array}$ \\
\hline Re-hospitalization & $27(13.4)$ & $8(11.9)$ & $11(34.4)$ & $21(39.6)$ & $<0.001$ \\
\hline \multicolumn{6}{|l|}{ Bayley-III } \\
\hline Cognitive & $\begin{array}{l}100(90- \\
110)\end{array}$ & $\begin{array}{l}95(90- \\
105)\end{array}$ & $95(90-102.5)$ & $90(80-95)$ & $<0.001$ \\
\hline Language & $97(89-109)$ & $\begin{array}{l}91(86- \\
109)\end{array}$ & $\begin{array}{l}95.5(81- \\
101.5)\end{array}$ & $\begin{array}{l}84.5(74- \\
94)\end{array}$ & $<0.001$ \\
\hline Motor & $\begin{array}{l}100(94- \\
107)\end{array}$ & $\begin{array}{l}97(88- \\
100)\end{array}$ & $\begin{array}{l}95.5(86.5- \\
100)\end{array}$ & $91(79-97)$ & $<0.001$ \\
\hline $\begin{array}{l}\text { Cognitive and Language } \\
<85\end{array}$ & $9(6.5)$ & $4(7.3)$ & $4(16.7)$ & $11(23.9)$ & 0.008 \\
\hline Motor $<85$ & $11(8)$ & $3(5.5)$ & $5(20.8)$ & $15(32.6)$ & $<0.001$ \\
\hline $\mathrm{CP}$ & $5(2.5)$ & $5(7.5)$ & $0(0)$ & $5(9.4)$ & 0.039 \\
\hline Blindness & - & - & - & - & \\
\hline Hearing aids & - & - & - & - & \\
\hline Combined NDI & $16(7.9)$ & 8 (11.9) & $5(15.6)$ & $21(39.6)$ & $<0.001$ \\
\hline
\end{tabular}

Multivariate logistic regression analysis was conducted to calculate the adjusted odds ratio (OR) for rehospitalization and combined NDI based on the three different criteria for BPD (Figure 2). The adjusted OR for re-hospitalization was highest in grade 3 in the NICHD 2018 (6.77, 95\% confidence interval (Cl) 2.05-22.36) and grade 3 in the Jensen (2019) criteria (7.45, 95\% Cl 1.49-37.33) (Figure 2(A)). Adjusted OR for combined NDI was highest in the severe BPD or Grade 3 BPD of all three criteria, while the adjusted OR was highest in the Grade 3 of the Jensen (2019) criteria (13.23, 95\% Cl 2.82-62.21) (Figure 2(B)). Multivariate analysis for PAH at a PMA of 36 weeks based on each definition showed that PAH was associated with Grade 2 and Grade 3 BPD of the NICHD 2018 and the Jensen criteria, with the highest adjusted OR in the Grade 3 of Jensen's (40.37, 95\% Cl 5.15-316.34) (Figure 3).

\section{Discussion}

In this study, the diagnostic criteria for BPD established in 2001, the newly proposed criteria in 2018, and the Jensen criteria in 2019 were used to define the severity of the disease considering the prediction of 
later health outcomes in preterm infants born at $<32$ weeks' gestation. While only the most severe diseases were associated with combined NDI in the NIH 2001 and the NICHD 2018 criteria, both Grade 2 and Grade 3 BPD in the Jensen (2019) criteria were associated with NDI. Both Grade 2 and Grade 3 BPD in the NICHD 2018 and the Jensen criteria were associated with PAH at a PMA of 36 weeks, with the highest adjusted OR in Grade 3 BPD in the Jensen criteria.

Changes in daily practices, including the use of HHFNC with room air (21\% oxygen) or very low flow with $100 \%$ oxygen, makes is difficult to classify many infants using the $\mathrm{NIH} 2001$ definition.[4] Furthermore, modalities that provide synchronized or non-synchronized NIPPV have also been increasingly used to improve post-extubation stabilization in preterm infants.[13]

The criteria of the NICHD in 2018 were proposed to reflect current practice in the neonatal intensive care unit through a review of various non-invasive breathing aids, and not only HHFNC but also various noninvasive modalities were considered and integrated to define the severity of the disease. Moreover, the NICHD 2018 criteria distinguish infants who required invasive ventilation and those who did not require invasive ventilation in the severe grade, as it is evident that infants who required protracted invasive ventilation had unfavorable respiratory and/or neurodevelopmental outcomes.[14] The criteria also excluded those who had airway problems without parenchymal lung disease and required at least 3 days of observation to determine the degree of respiratory support.

Jensen et al compared various pre-specified definitions of BPD according to respiratory support and supplemental oxygen levels at a PMA of 36 weeks in preterm infants born at $<32$ weeks' gestation. [8] The definition with the best predictability was found when BPD was graded according to the mode of respiratory support regardless of oxygen use. Jensen et al. 's findings are intriguing, as we previously reported that higher capillary partial pressure of carbon dioxide, rather than the requirement for oxygen, was a good predictor of later respiratory outcomes among patients with severe BPD without invasive ventilation at a PMA of 36 weeks.[15]

Better prediction of later outcomes in the Jensen (2019) criteria seem to be obvious, as it was selected based on its predictive ability. A notable finding of the current study is that the association of PAH at a PMA of 36 weeks was also well demonstrated in the newer criteria, especially in the Jensen (2019) criteria. BPD is now recognized as a form of pulmonary vascular disease in premature infants, abnormalities in the development of pulmonary blood vessels, along with lung damage, could lead to impairment in the structure and function of pulmonary blood vessels.[16] PAH is a spectrum of vascular disease, which is frequently accompanied by moderate to severe BPD and is associated with poor outcomes in preterm infants.[10] Therefore, the Jensen (2019) criteria might discriminate more severe diseases and reflects the pathophysiology of chronic lung disease in prematurity compared to previous criteria.

There are several limitations to this study that should be acknowledged. First, this was a single-center study; therefore, the number of subjects was small compared to large-scale studies.[8; 17] Second, only $74.3 \%$ of the study population was tested using Bayley-III at a CA of 18-24 months, although other 
neurodevelopmental outcomes, such as $\mathrm{CP}$, hearing impairment, and blindness have been well reported. However, other aspects of respiratory outcomes, such as respiratory medication and respiratory symptoms, were not assessed. Furthermore, socioeconomic and environmental factors that influence respiratory and neurodevelopmental outcomes were not included in this study.

\section{Conclusions}

Recently suggested BPD criteria, such as the NICHD 2018 and Jensen's (2019) criteria, showed an association between the severity of BPD and later outcomes in preterm infants. Furthermore, the association of BPD severity based on these recent criteria and PAH was well demonstrated in this study. As PAH is an important aspect of the pathophysiology of BPD, it might be speculated that recent criteria of BPD could discriminate severity of disease not only more practically, but also more based on the entity of BPD.

\section{Abbreviations}

Bayley-III: Bayley scales of infant and toddler development 3rd edition

BPD: Bronchopulmonary dysplasia

CA: Corrected age

Cl: Confidence interval

HHFNC: Humidified high flow nasal cannula

NDI: Neurodevelopmental impairment

NICHD: National institute of child health and human development

$\mathrm{NIH}$ : National institutes of health

NIPPV: Non-invasive positive pressure ventilation

OR: Odds ratio

PAH: Pulmonary arterial hypertension

PMA: Postmenstrual age

\section{Declarations}

Funding: The authors declare that no funds, grants, or other support were received during the preparation of this manuscript. 
Conflicts of interest/Competing interests: The authors have no relevant financial or non-financial interests to disclose.

Availability of data and material: Not applicable.

Code availability: Not applicable.

\section{Authors' contributions:}

Conceptualization: Jae Kyoon Hwang, Seung Han Shin

Data curation: Jae Kyoon Hwang, Seung Han Shin

Formal Analysis: Jae Kyoon Hwang, Seung Han Shin

Investigation: Jae Kyoon Hwang, Seh Hyun Kim

Methodology: Seung Han Shin, Ee-Kyung Kim, Seh Hyun Kim

Software: Seung Han Shin

Supervision: Seung Han Shin, Han-Suk Kim

Validation: Seung Han Shin, Ee-Kyung Kim, Han-Suk Kim

Visualization: Seung Han Shin, Ee-Kyung Kim

Writing - original draft: Jae Kyoon Hwang, Seung Han Shin, Seh Hyun Kim

Writing - review \& editing: Jae Kyoon Hwang, Seung Han Shin, Han-Suk Kim

Ethics approval: This study was approved by the Institutional Review Board of Seoul National University Hospital (2109-008-1250).

Consent to participate: Obtaining informed consent was waived by the board and all methods were performed in accordance with the guidelines of the Human Research Protection Program.

Consent for publication: Not applicable.

\section{References}

1. Northway WH Jr, Rosan RC, Porter DY (1967) Pulmonary disease following respirator therapy of hyaline-membrane disease. Bronchopulmonary dysplasia.N Engl J Med276:357-368

2. Jobe AH, Bancalari E (2001) Bronchopulmonary dysplasia.Am J Respir Crit Care Med163:17231729 
3. Walsh MC, Wilson-Costello D, Zadell A, Newman N, Fanaroff A (2003) Safety, reliability, and validity of a physiologic definition of bronchopulmonary dysplasia.J Perinatol23:451-456

4. Poindexter BB, Feng R, Schmidt B, Aschner JL, Ballard RA, Hamvas A, Reynolds AM, Shaw PA, Jobe AH, Prematurity P (2015) Comparisons and Limitations of Current Definitions of Bronchopulmonary Dysplasia for the Prematurity and Respiratory Outcomes Program. Ann Am Thorac Soc 12:18221830

5. Sand L, Szatkowski L, Kwok TnC, Sharkey D, Todd DA, Budge H, Ojha S (2021) Observational cohort study of changing trends in non-invasive ventilation in very preterm infants and associations with clinical outcomes. Archives of Disease in Childhood - Fetal and Neonatal Edition:fetalneonatal-2021322390

6. Weisz DE, Yoon E, Dunn M, Emberley J, Mukerji A, Read B, Shah PS (2021) Duration of and trends in respiratory support among extremely preterm infants.Archives of Disease in Childhood - Fetal and Neonatal Edition106:286-291

7. Higgins RD, Jobe AH, Koso-Thomas M, Bancalari E, Viscardi RM, Hartert TV, Ryan RM, Kallapur SG, Steinhorn RH, Konduri GG, Davis SD, Thebaud B, Clyman RI, Collaco JM, Martin CR, Woods JC, Finer NN, Raju TNK (2018) Bronchopulmonary Dysplasia: Executive Summary of a Workshop.J Pediatr197:300-308

8. Jensen EA, Dysart K, Gantz MG, McDonald S, Bamat NA, Keszler M, Kirpalani H, Laughon MM, Poindexter BB, Duncan AF, Yoder BA, Eichenwald EC, DeMauro SB (2019) The Diagnosis of Bronchopulmonary Dysplasia in Very Preterm Infants. An Evidence-based Approach.Am J Respir Crit Care Med200:751-759

9. Mourani PM, Sontag MK, Younoszai A, Miller JI, Kinsella JP, Baker CD, Poindexter BB, Ingram DA, Abman SH (2015) Early Pulmonary Vascular Disease in Preterm Infants at Risk for Bronchopulmonary Dysplasia.Am J Resp Crit Care191:87-95

10. Hansmann G, Sallmon H, Roehr CC, Kourembanas S, Austin ED, Koestenberger M, European Pediatric Pulmonary Vascular Disease N (2020) Pulmonary hypertension in bronchopulmonary dysplasia. Pediatr Res

11. Choi EK, Shin SH, Kim EK, Kim HS (2019) Developmental outcomes of preterm infants with bronchopulmonary dysplasia-associated pulmonary hypertension at 18-24 months of corrected age.BMC Pediatr19:26

12. Johnson S, Moore T, Marlow N (2014) Using the Bayley-III to assess neurodevelopmental delay: which cut-off should be used?Pediatr Res75:670-674

13. Ferguson KN, Roberts CT, Manley BJ, Davis PG (2017) Interventions to Improve Rates of Successful Extubation in Preterm Infants: A Systematic Review and Meta-analysis.JAMA Pediatr171:165-174

14. Choi SH, Shin SH, Kim E-K, Kim H-S (2019) Respiratory Outcomes at 12 Months of Corrected Age of Preterm Infants with Severe Bronchopulmonary Dysplasia Requiring Protracted Invasive Ventilation.Perinatology30:20-26 
15. Shin SH, Shin JS, Kim EK, Kim HS (2021) Capillary partial pressure of carbon dioxide for predicting rehospitalization in preterm infants under noninvasive respiratory support with severe bronchopulmonary dysplasia. Pediatr Pulmonol

16. Thebaud B, Goss KN, Laughon M, Whitsett JA, Abman SH, Steinhorn RH, Aschner JL, Davis PG, McGrath-Morrow SA, Soll RF, Jobe AH (2019) Bronchopulmonary dysplasia.Nat Rev Dis Primers5:78

17. Isayama T, Lee SK, Yang J, Lee D, Daspal S, Dunn M, Shah PS, Canadian Neonatal N, Canadian Neonatal Follow-Up Network I (2017) Revisiting the Definition of Bronchopulmonary Dysplasia: Effect of Changing Panoply of Respiratory Support for Preterm Neonates.JAMA Pediatr171:271-279

\section{Figures}

\section{GA $<32$ weeks $(2014 \sim 2018)$ $(n=479)$}

\section{Congenital anomaly $(\mathrm{n}=19)$ Congenital infection $(\mathrm{n}=1)$ Death before discharge $(n=60)$}

\section{Lost to FU at CA 18 24 months $(\mathrm{n}=45)$}

\section{Survival to discharge $(\mathrm{n}=399)$}

\section{Infants included during analysis $(\mathrm{n}=354)$}

\section{Figure 1}

Flow chart of study population CA, corrected age; FU, follow up; GA, gestational age 

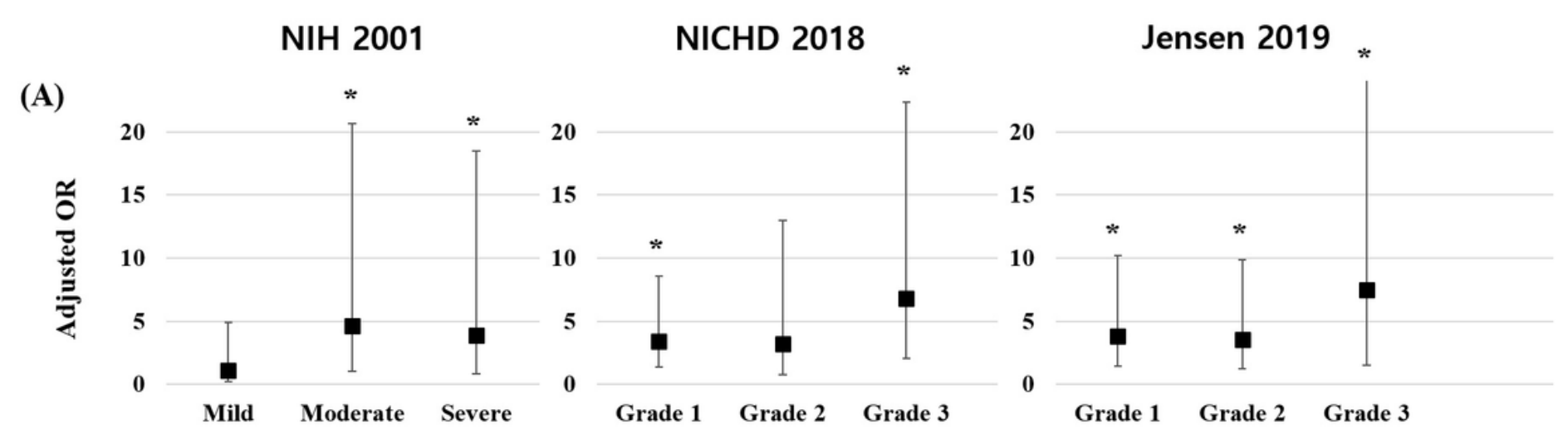

(B)
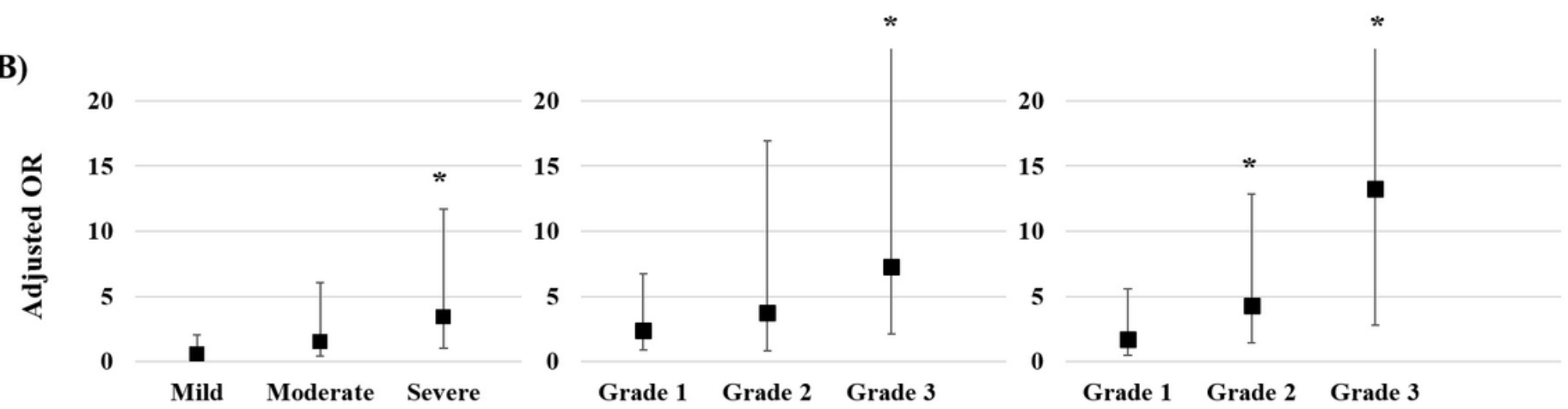

Figure 2

Multivariate analysis for respiratory and neurodevelopmental outcomes at a CA of 18-24 months based on each of the criteria for BPD. Gestational age, birthweight z-score, and sex were adjusted in the multivariate analysis. Adjusted OR of the severity of BPD based on the three criteria for re-hospitalization due to respiratory illness until a CA of 18-24 months (A). Adjusted OR of the severity of BPD based on the three criteria for NDI at a CA of 18-24 months (B). CA, corrected age; BPD, bronchopulmonary dysplasia; OR, odds ratio; NDI, neurodevelopmental impairment; NIH, National Institutes of Health; NICHD, National Institute of Child Health and Human Development

NIH 2001

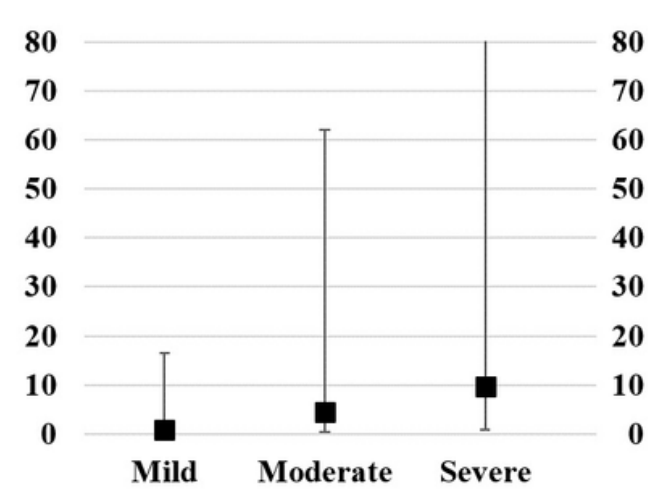

NICHD 2018

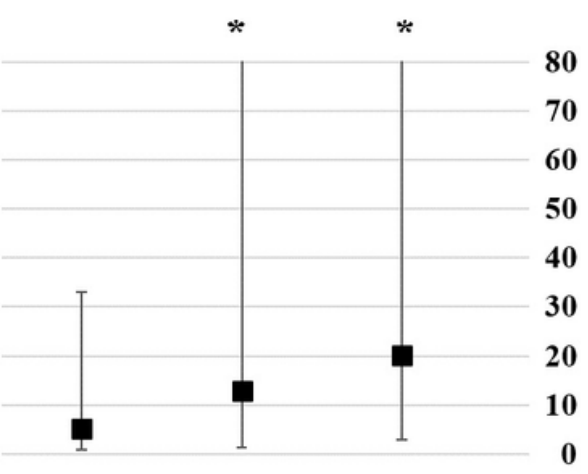

Grade 1 Grade 2 Grade 3
Jensen 2019

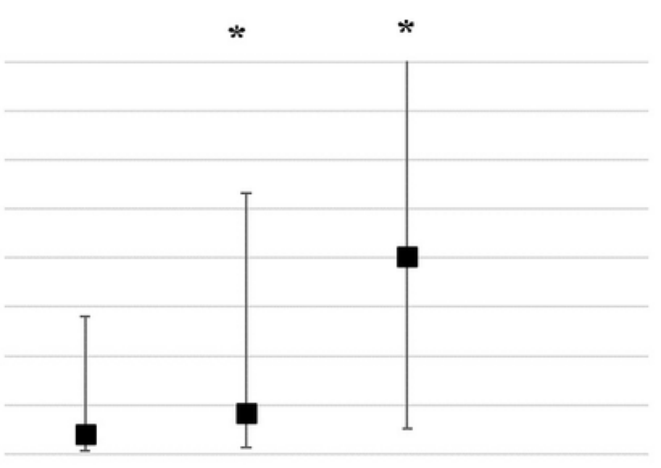

Grade 1 Grade 2 Grade 3

Figure 3 
Adjusted OR for PAH at a PMA of 36 weeks based on each of the criteria for BPD. Gestational age, birthweight z-score, and sex were adjusted in the multivariate analysis. OR, odds ratio; PAH, pulmonary arterial hypertension; PMA, postmenstrual age; BPD, bronchopulmonary dysplasia.

\section{Supplementary Files}

This is a list of supplementary files associated with this preprint. Click to download.

- BPDSupplementarytable.docx 